\title{
Entendendo os Relacionamentos Íntimos com Comportamento Abusivo por meio da Teoria do Apegod
}

\author{
Amanda de Souza $\operatorname{Tosta}^{1}{ }^{1}$, \& Vicent Cassepp-Borges ${ }^{(D)}{ }^{2}$
}

Universidade Federal Fluminense, Rio de Janeiro, Brasil.

\begin{abstract}
RESUMO
Este trabalho tem como objetivo avaliar a validade das escalas ARI que evidenciam e correlacionam os tipos de violências vividas dentro de um relacionamento íntimo (Física, Sexual, Verbal e Psicológica), além de avaliar diferenças de gênero na violência conjugal sofrida e praticada, bem como a relação desta escala com os tipos de apego. Participaram desse estudo 464 mulheres e 85 Homens, respondendo à Experiencie in Close Relationships (ECR), à Escala de Abuso em Relacionamento Íntimos (ARI) e Abuso em Relacionamento Íntimos baseado no comportamento do respondente em relação ao outro (ARI-E) e um questionário demográfico por meio eletrônico. Os resultados indicaram que as escalas ARI e ECR apresentam boas propriedades psicométricas. Com relação à violência, as mulheres relataram sofrer significativamente mais com violência física, enquanto homens relataram sofrer mais com violência psicológica e atitudes controladoras. Esse estudo sugere que, a escala ARI tem bons indicadores de validade e confiabilidade na avaliação da violência em relacionamentos íntimos.
\end{abstract}

Palavras-chave

relacionamentos abusivos; teoria do apego; vinculação

\begin{abstract}
This work aims to evaluate the validity of the scales ARI that show and correlate the types of violence experienced within an intimate relationship (Physical, Sexual, Verbal and Psychological), in addition to evaluating gender differences in the conjugal violence suffered and practiced, as well as the relation of this scale with the types of attachment. 464 women and 85 men participated in this study, responding to the Experiences in Close Relationships (ECR), the Scale of Abuse in Intimate Relationships (ARI) and Abuse in Intimate Relationships based on the behavior of the respondent in relation to the other (ARI-E) and one demographic questionnaire applied electronically. The results indicated that the ARI and ECR scales have good psychometric properties. Regarding the violence, women reported suffering significantly more from physical violence, while men reported suffering more from psychological violence and controlling attitudes. This study suggests that the ARI scales has good indicators of validity and reliability in the assessment of violence in intimate relationships.
\end{abstract}

Keywords

abusive relationships; attachment; attachment theory

\footnotetext{
${ }^{1}$ Correspondence about this article should be addressed to Amanda de Souza Tosta: amandastosta@ gmail.com

${ }^{2}$ Conflicts of Interest: The authors declare that the research was conducted in the absence of any commercial or financial relationships that could be construed as a potential conflict of interest.
} 


\section{Understanding Intimate Relationships with Abusive Behavior through Attachment Theory}

\section{Introdução}

É possível qualificar a violência como um fato cotidiano e universal, que sobreexiste desde os primórdios das civilizações. Como qualquer conceito relacionado a processos culturais amplos, carrega uma carga semântica rica, cuja complexidade é expressão da própria variedade social humana. Para Piosiadlo, Fonseca e Gessner (2014), embora exista um consenso razoável a respeito do que seja a violência, o contexto temporal é determinante no grau de aceitação das suas variadas modalidades. A Organização Mundial de Saúde (OMS) define a violência como ação que tem como resultado possível de pior intensidade a morte e em gradações menores lesões físicas, comprometimento do desenvolvimento do indivíduo que a sofre ou dano psicológico, ação empreendida pelo uso da força física ou do poder, na forma de ameaça ou de violência atual, de maneira intencional - contra si ou outra pessoa, contra comunidades ou agrupamentos (Krug, Dahlberg, Mercy, Zwi. \& Lozano, 2002).

Paiva e Figueiredo (2004, p. 244) julgam que o "conhecimento empírico é consensual quanto à presença de algum índice de disfunção ou de abuso no contexto das relações íntimas". As pesquisas sobre a violência em relacionamentos íntimos de adolescentes são bem numerosas no mundo anglo-saxão (Cornelius \& Resseguie, 2007, Hickman, Jaycox, \& Aronoff, 2004, Ting, 2009), o que talvez seja indicativo tanto do destaque social dado ao tema na última década quanto da gravidade das suas consequências. Um exame rápido dos estudos de Barnett, Miller-Perrin e Perrin (1997) ou Henderson, Bartholomew, Trinke e Kwong (2005) revela que o abuso em relacionamento íntimo no começo da vida adulta é, pelo menos, frequente. Nos Estados Unidos da América (EUA), 10\% das meninas de 12 a 15 anos e 22\% das garotas entre 16 e 19 anos foram mortas entre os anos de 1993 e 1999, vítimas de seus namorados (Murta, Santos, Martins, \& Oliveira, 2013).

Cerca de $87 \%$ de mais de 3000 jovens adultos de dez estados brasileiros entrevistados já foram vítimas de algum tipo de abuso (Oliveira, Assis, Njaine, \& Oliveira, 2011). Para Safiotti (2004), provavelmente todas as mulheres já foram vítimas de algum tipo de violência de gênero, ainda que não denominem dessa forma. As várias modalidades do abuso (físico, psicológico, verbal, sexual) acontecem de maneira interrelacionada na maior parte das vezes (Aldrighi, 2004, Anacona, 2008). O abuso é, 
também, como já mencionado, bidirecional e multicausal (Williams, Ghandour, \& Kub, 2008), proveniente de elementos familiares, culturais e pessoais - ter sofrido abuso sexual ou conviver em comunidades pronunciadamente sexistas (Caridade \& Machado, 2006, Dilillo, Giuffre, Tremblay, \& Peterson, 2001), ter testemunhado repetidas vezes o abuso entre os próprios pais ou familiares próximos ou ser portador de problemas graves de controle emocional (Anacona, 2008; Ting, 2009), entre outros.

A violência em relacionamentos íntimos é uma questão de saúde, já que suas implicações na saúde física e mental das pessoas envolvidas não pode ser ignorada. Interessa-nos aqui, sobretudo o abuso no relacionamento íntimo como um fenômeno que influi sobre o quadro da saúde mental dos indivíduos. Entre as consequências psicológicas mais graves do abuso estão o estresse pós-traumático, a queda na autoestima e a ideação suicida, todos prevalentes no sexo feminino (Caridade \& Machado, 2006). Um efeito grave da violência nos relacionamentos íntimos é que a violência iniciada na fase de namoro acabe em diversos casos por ser determinante do padrão de comportamento na coabitação matrimonial (Chan, Straus, Brownridge, Tiwari, \& Leung, 2008). É fundamental considerar que o namoro é o momento da relação em que são construídas as relações conjugais futuras. Assim sendo, é possível que o namoro possa servir como um fator decisivo para prevenção da violência nos relacionamentos íntimos (Flake, Barros, Schraiber, \& Menezes, 2013). Até a década de 1980, porém, a maior parte dos estudos lidava apenas com a violência conjugal. A partir de então, o seu escopo tem sido ampliado para o namoro e até mesmo a intimidade de um tipo mais casual. Há estudos importantes sobre a violência em relacionamentos íntimos na fase escolar, que indicam que de 15 a $43 \%$ da violência vivida por jovens nesta fase ocorrem dentro das dependências escolares (Flake et al., 2013).

Segundo Paiva e Figueiredo (2003), o relacionamento abusivo é caracterizado pela recorrência significativa de atos de violência. Não é um tema "novo", e remete a muitos anos de uma história de opressões de gênero e de sexualidade, em especial às mulheres. O abuso no relacionamento íntimo tem efeitos nocivos na qualidade de vida, na saúde mental e física da vítima. Algumas pesquisas (Caridade \& Machado, 2006; Matos, 2006; Paiva \& Figueiredo, 2003) indicam ser cada vez mais frequente o comportamento abusivo dentro dos relacionamentos íntimos. É sabido que homens, embora em uma escala muito menor, também sofrem com abuso em relacionamentos íntimos (Hohendorf, Habigzang, \& Koller, 2017), mas a literatura científica apresenta mais dados sobre as mulheres (Hohendorff, Santos \& Dell'Aglio, 2015). É essencial 
realizar uma problematização acerca das bases materiais, subjetivas e sociais que sustentam essa realidade cruel e tão naturalizada pela ideologia dominante. Saffioti (2004) ressalta que nos últimos milênios da história, as mulheres estiveram hierarquicamente inferiores aos homens. "Tratar esta realidade exclusivamente em termos de gênero distrai a atenção do poder do patriarca, em especial como homem/marido, 'neutralizando' a exploração-dominação masculina"

A teoria do apego/vinculação afetiva adulta de Hazan e Shaver (1987) apresenta possibilidades de ser usada como chave de leitura do fenômeno do abuso nos relacionamentos íntimos. Baseadas nas experiências e nos padrões típicos de interação com as figuras significativas durante a infância, cada pessoa eventualmente constrói "modelos internos dinâmicos" que irão guiar os seus comportamentos interpessoais posteriores (Paiva \& Figueiredo, 2003). É a partir desses modelos que a mesma estabelece expectativas acerca do que pode esperar de si e dos outros em relacionamentos.

Apego diz respeito ao vínculo afetivo/emocional estabelecido entre seres humanos, em que um deles representa uma figura de apego e o outro busca proximidade a essa figura em situações de desconforto ou ameaça (Natividade \& Shiramizu, 2015). Conforme observa Golse (1998 em Dalbem \& Dell Áglio, 2005), o comportamento de apego não é algo herdado, ele se desenvolve ao longo da vida e possui bases instintivas, o que é herdado é a capacidade de adaptação para evolução e preservação presentes num tipo de código genético. Há ainda evidências que o comportamento de apego não é sustentado apenas por associações de prazer, pois crianças por vezes também se apegam a modelos abusivos.

Hazan e Shaver (1987) perceberam que indivíduos com um estilo de apego seguro percebiam a relação com o parceiro como mais afetuosa, amigável e confiante. Já indivíduos com estilos de apego inseguro ansioso (que remete a um cuidado inconsistente durante a infância) relataram uma maior necessidade de desejo por união e reciprocidade com o parceiro romântico. Por último, indivíduos apresentando um estilo de apego inseguro evitativo (associado com um cuidado insensível durante a infância) relataram sentir-se incomodados com a proximidade física e emocional com o parceiro romântico.

Apesar da importância do tema da violência conjugal para a sociedade, são escassos os instrumentos para a medida do fenômeno. Este trabalho tem como objetivo avaliar a validade das escalas (ARI) que evidenciam e correlacionam os tipos de violências vividas dentro de um relacionamento íntimo (Física, Sexual, Verbal e 
Psicológica), além de avaliar diferenças de gênero na violência conjugal sofrida e praticada, bem como a relação desta escala com os tipos de apego.

\section{Método}

\section{Participantes}

A pesquisa teve a participação de 549 pessoas, sendo 85 homens $(15,5 \%)$ e 464 mulheres $(84,5 \%)$. A idade dos participantes foi de 14 e 62 anos, com uma média de 25,2 anos $(D P=7,4)$. A amostra foi composta por uma maioria de heterossexuais $(n=440$, $80,1 \%)$, mas com um considerável número de bissexuais $(n=87,15,8 \%)$ e homossexuais $(n=22,4,0 \%)$. Mais da metade $(n=326,59,4 \%)$ eram estudantes. As principais religiões encontradas foram Catolicismo $(n=167,30,4 \%)$ e Espiritismo $(n=63,11,5 \%)$, sendo que um considerável número se declarou sem religião $(n=118,21,5 \%)$ ou Ateu/Agnóstico $(n=79,14,4 \%)$. Quanto ao status de relacionamento no momento da realização da coleta de dados, a maioria namora ou já namorou $(n=411,74,9 \%)$, seguido de casados $(n=82$, $14,9 \%)$, relacionamentos sem compromisso $(n=41,7,5 \%)$ e divorciados/viúvos $(n=13$, $2,4 \%)$.

\section{Instrumentos}

Foi aplicado um protocolo dividido em quatro partes, que serão descritas na ordem em que foram apresentadas aos participantes. A primeira foi um questionário demográfico; a segunda foi uma adaptação de um teste disponível no website norte americano de apoio a vítimas de abuso em relacionamento íntimo Love is Respect (2016). Essa adaptação deu origem a Escala de Abuso em Relacionamento Íntimos (ARI) que se subdivide em Abuso em Relacionamento Íntimos baseado no comportamento do outro em relação ao respondente (ARI-O) e Abuso em Relacionamento Íntimos baseado no comportamento do respondente em relação ao outro (ARI-E). A terceira está baseada no trabalho de Natividade e Shiramizu (2015), que se propõe aferir apego adulto através do instrumento traduzido e adaptado para o Brasil: Experience in Close Relationships Inventory (ECR) (Brennan, Clark, \& Shaver, 1998). A quarta e última parte, uma questão aberta para relatar experiências em relacionamento abusivo.

No questionário, as dimensões evitação e ansiedade, foram inferidos a partir de 10 afirmações nas quais os participantes responderam em uma escala de sete pontos o quanto concordavam com os itens, em que o ponto 1 corresponde a 'discordo fortemente' 
e o 7, a 'concordo fortemente' para a escala ECR adaptada e reduzida para o Brasil (Natividade \& Shiramizu, 2015). O ARI possui 25 afirmações cada, nas quais o respondente teria que em uma escala de cinco pontos variando em "nunca", "raramente", "às vezes", "frequentemente" e "sempre" assinalar a frequência que os comportamentos acontecem no relacionamento. Não foram encontrados estudos psicométricos anteriores ao presente estudo com a escala ARI.

\section{Procedimentos}

As coletas de dados foram realizadas de modo individual, por meio da aplicação de um questionário online na plataforma Googleforms ${ }^{\circledR}$, sobre o qual os participantes foram instruídos pela pesquisadora. O questionário foi divulgado em grupos de redes sociais e sites de vítimas de abuso, estudantes universitários e via aplicativos de mensagens por telefone celular. Os participantes também foram solicitados a compartilhar o questionário em seu círculo de amizades (método da bola de neve). $\mathrm{O}$ questionário ficou disponibilizado durante três meses (setembro/2016 - novembro/2016).

Os procedimentos éticos, conforme dispostos na resolução 510/2016 do Conselho Nacional de Saúde, foram cuidadosamente observados. Os participantes foram informados por meio de um Termo de Consentimento Livre e Esclarecido sobre as condições de estudo e selecionaram a opção concordando com a sua participação no estudo. Embora a pesquisa tenha sido direcionada a maiores de 18 anos, poucos participantes ( $n=16,2,9 \%)$ declararam ser menores de idade. Optou-se por mantê-los na amostra.

A relação entre apego e no nível de abuso também foi analisada por correlação de Pearson. As médias de homens e mulheres foram comparadas por meio de teste $t$ de Student. Os dados foram analisados por meio de análise fatorial exploratória com extração Principal Axis Factoring e rotação oblíqua Direct Oblimin para todas as escalas exceto para a escala ARI-E na qual foi realizada uma análise fatorial confirmatória. Foram calculados os valores de alfa de Cronbach para avaliar a consistência interna dos instrumentos e seus respectivos fatores.

\section{Resultados}

O primeiro passo foi realizar uma análise exploratória da escala ARI-O. Observou-se que a escala ARI-O teve um Kaiser-Meyer-Olkin (KMO) de 0,95 e um Teste 
de Esfericidade de Bartlett significativo $\left(\chi^{2}=9880,3 ; g l=300\right.$ e $\left.p<0,001\right)$. Esses valores indicam uma boa fatorabilidade da matriz de correlações. A partir do scree plot (Figura1), é possível inferir, com certa segurança, que a escala tem três fatores. A análise paralela também sugere a estrutura com 3 fatores. Estes fatores foram denominados: violência física, violência psicológica e atitudes controladoras. A Tabela 1 apresenta a carga fatorial de cada item em seu respectivo fator que, em geral, são elevadas

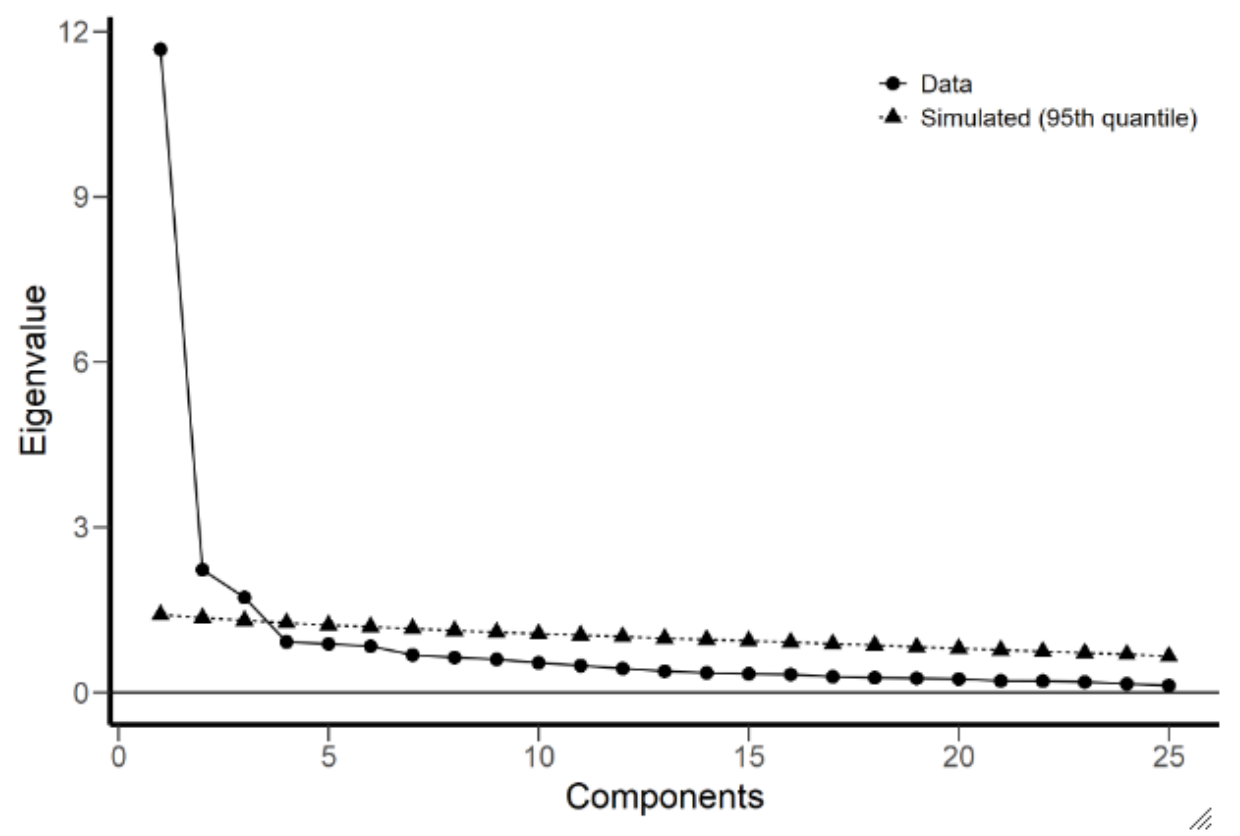

Figura 1. Scree plot da ARI-O

Para fazer a avaliação da escala ARI-E, foi realizada uma análise fatorial confirmatória, a partir do modelo encontrado na análise fatorial exploratória da ARI-O (que foi retestado em uma Análise Fatorial Confirmatória). Foi testado, ainda, um modelo alternativo com todos os itens carregando em um único fator. Pode-se perceber, na comparação das estruturas da ARI-O com a ARI-E, que o CFI e o TLI pioraram, mas o RMSEA permaneceu praticamente igual. $\mathrm{O} \chi^{2}$, entretanto, apresentou um menor desajuste (Tabela 2). Também se comparou uma solução da escala ARI-E com um fator só, piorando bastante todos os índices de ajustes, o que demonstra que essa solução com um fator não obteve nenhum suporte empírico. Embora não tenha repetido os índices de ajuste da escala ARI-O, a solução com três fatores da ARI-E tem um ajuste melhor do que a solução com um fator. Para manter a comparabilidade das estruturas da ARI-O com a ARI-E, optou-se pela escala com três fatores, que obteve um maior suporte empírico que a solução com um fator. 
Tabela 1.

Carga Pattern dos itens nos fatores da ARI-O

\begin{tabular}{cccc}
\hline Item & $\begin{array}{c}\text { Violência } \\
\text { Psicológica }\end{array}$ & $\begin{array}{c}\text { Violência } \\
\text { Física }\end{array}$ & Atitudes Controladoras \\
\hline O-3 & $-0,932$ & & \\
O-1 & $-0,865$ & & \\
O-2 & $-0,823$ & & \\
O-4 & $-0,695$ & & \\
O-15 & 0,645 & & \\
O-16 & 0,639 & & \\
O-21 & 0,619 & & \\
O-17 & 0,534 & & \\
O-5 & 0,531 & & \\
O-14 & 0,498 & 0,884 & 0,437 \\
O-23 & & 0,830 & 0,304 \\
O-20 & & 0,821 & \\
O-22 & & 0,791 & 0,6787 \\
O-18 & & 0,562 & \\
O-24 & & 0,408 & \\
O-25 & & 0,405 & \\
O-19 & & & \\
O-9 & 0,344 & & \\
O-7 & & & \\
O-12 & 0,410 & & \\
O-10 & 0,427 & & \\
O-11 & 0,320 & & \\
O-13 & & & \\
O-6 & & & \\
O-8 & & & \\
\hline
\end{tabular}

Nota: Método de extração: Principal Axis Factoring. Método de rotação: Oblimin com normalização de Kaiser. A rotação convergiu em 9 interações.

Tabela 2.

Análise Fatorial confirmatória da ARI-O e da ARI-E

\begin{tabular}{cccc}
\hline & ARI-O & ARI-E_3F & ARI-E_1F \\
\hline RMSEA & 0,095 & 0,091 & 0,111 \\
TLI & 0,848 & 0,691 & 0,545 \\
CFI & 0,862 & 0,72 & 0,583 \\
$\chi^{2}$ & 1620 & 1511 & 4727 \\
Gl & 272 & 272 & 300 \\
\hline
\end{tabular}


A Tabela 3 compara a carga fatorial da ARI-O com a ARI-E na análise fatorial confirmatória. Na referida Tabela, pode-se analisar item por item para a verificação de quais são os itens que na escala ARI-O de fato carregaram o fator e que na escala ARI-E tiveram uma carga bem menor. Assim, é provável que os participantes tenham interpretado aquele item como sendo de algum outro fator, e não como sendo um item associado naquele fator: seja violência psicológica, física ou atitudes controladoras.

Tabela 3.

Comparação da carga fatorial da ARI-O e ARI-E

\begin{tabular}{|c|c|c|c|c|c|c|c|}
\hline \multicolumn{5}{|c|}{ ARI-O } & \multicolumn{3}{|c|}{ ARI-E } \\
\hline Item & Estimativa & $\begin{array}{c}\text { Erro } \\
\text { Padrão }\end{array}$ & $\mathrm{p}$ & Item & Estimativa & $\begin{array}{c}\text { Erro } \\
\text { Padrão }\end{array}$ & $\mathrm{p}$ \\
\hline Psicológica & & & & Psicológica & & & \\
\hline $\mathrm{O}-3$ & 0,683 & 0,024 & 0,000 & E-3 & 0,301 & 0,044 & 0,000 \\
\hline O-1 & 0,721 & 0,022 & 0,000 & E-1 & 0,236 & 0,045 & 0,000 \\
\hline $\mathrm{O}-2$ & 0,702 & 0,023 & 0,000 & E-2 & 0,177 & 0,046 & 0,000 \\
\hline O-4 & 0,747 & 0,020 & 0,000 & E-4 & 0,351 & 0,043 & 0,000 \\
\hline O-15 & $-0,883$ & 0,011 & 0,000 & E-15 & $-0,719$ & 0,027 & 0,000 \\
\hline O-16 & $-0,864$ & 0,012 & 0,000 & E-16 & $-0,696$ & 0,028 & 0,000 \\
\hline O-21 & $-0,846$ & 0,013 & 0,000 & E-21 & $-0,523$ & 0,036 & 0,000 \\
\hline O-17 & $-0,768$ & 0,019 & 0,000 & E-17 & $-0,627$ & 0,032 & 0,000 \\
\hline O-5 & $-0,692$ & 0,023 & 0,000 & E-5 & $-0,259$ & 0,044 & 0,000 \\
\hline O-14 & $-0,798$ & 0,017 & 0,000 & E-14 & $-0,604$ & 0,032 & 0,000 \\
\hline Física & & & & Física & & & \\
\hline $\mathrm{O}-23$ & 0,789 & 0,019 & 0,000 & E-23 & 0,722 & 0,023 & 0,000 \\
\hline O-20 & 0,792 & 0,019 & 0,000 & E-20 & 0,788 & 0,020 & 0,000 \\
\hline O-22 & 0,822 & 0,017 & 0,000 & E-22 & 0,742 & 0,022 & 0,000 \\
\hline O-18 & 0,763 & 0,021 & 0,000 & E-18 & 0,858 & 0,015 & 0,000 \\
\hline O-24 & 0,726 & 0,023 & 0,000 & E-24 & 0,483 & 0,036 & 0,000 \\
\hline O-25 & 0,551 & 0,032 & 0,000 & E-25 & 0,350 & 0,040 & 0,000 \\
\hline O-19 & 0,541 & 0,033 & 0,000 & E-19 & 0,670 & 0,026 & 0,000 \\
\hline $\begin{array}{c}\text { Atitudes } \\
\text { Controladoras }\end{array}$ & & & & $\begin{array}{c}\text { Atitudes } \\
\text { Controladoras }\end{array}$ & & & \\
\hline O-9 & 0,848 & 0,014 & 0,000 & E-9 & 0,661 & 0,031 & 0,000 \\
\hline O-7 & 0,390 & 0,038 & 0,000 & E-7 & 0,297 & 0,044 & 0,000 \\
\hline O-12 & 0,920 & 0,009 & 0,000 & E-12 & 0,784 & 0,026 & 0,000 \\
\hline O-10 & 0,825 & 0,015 & 0,000 & E-10 & 0,616 & 0,033 & 0,000 \\
\hline O-11 & 0,793 & 0,017 & 0,000 & E-11 & 0,374 & 0,042 & 0,000 \\
\hline O-13 & 0,812 & 0,016 & 0,000 & E-13 & 0,532 & 0,037 & 0,000 \\
\hline O-6 & 0,290 & 0,040 & 0,000 & E-6 & 0,161 & 0,046 & 0,001 \\
\hline O-8 & 0,503 & 0,033 & 0,000 & E-8 & 0,178 & 0,046 & 0,000 \\
\hline
\end{tabular}


Os valores de alpha de Cronbach foram muito bons em todos os fatores da escala ARI-O: Violência Física $(\alpha=0,86)$, Violência Psicológica $(\alpha=0,87)$ e Atitudes Controladoras $(\alpha=0,94)$. Com relação à escala ARI-E, os indicadores de confiabilidade foram menores, mas ainda assim, considerados aceitáveis: Violência Física $(\alpha=0,83)$, Atitudes Controladoras $(\alpha=0,71)$, Violência Psicológica $(\alpha=0,64)$. Desse modo, juntouse todos os itens de todas as escalas e criou-se uma média de todos os itens que compõem cada fator, obtendo um score do fator. Foi feito um escore para evitação e um para ansiedade, na ECR; um para violência psicológica, um para violência física e outro para atitudes controladoras, nas escalas ARI-E e ARI-O.

Comparou-se, na Tabela 4, os escores dos fatores de homens e mulheres, para que se pudesse descobrir diferenças de sexo com relação a cada tipo de violência e estilos de apego. Fez-se a comparação de médias com o Teste t de Student. Observa-se então, que o estilo de apego evitativo foi mais comum em homens, enquanto as mulheres apresentaram escores significativamente mais altos no apego ansioso. Quanto aos tipos de violência, não foi encontrada diferença significativa na escala ARI-E, ou seja, os respondentes não diferem em gênero na violência que admitem cometer, mesmo que tenha havido uma tendência à significância na ARI-E Atitudes Controladoras. No que tange a ARI-O, foi encontrada uma diferença de médias de gênero nas três sub-escalas. Os homens participantes da pesquisa alegam perceber que sofrem significativamente mais atitudes controladoras e violência psicológica, enquanto as mulheres participantes da pesquisa apontam sofrer significativamente mais com as violências físicas. Ressalta-se que esses são apenas dados médios da percepção dos(as) participantes, que podem não refletir a realidade de relações individuais.

Observando o fator atitudes controladoras, verificou-se que as médias dos homens na ARI-E é maior do que na ARI-O. Assim, eles relatam praticar mais atitudes controladoras do que sofrer. $\mathrm{O}$ mesmo fato pode ser observado nas mulheres nos fatores atitudes controladoras e violência psicológica. Ressalta-se que, com relação à violência psicológica, menores escores indicam maior nível de violência. Isso significa que os homens se percebem mais frequentemente como vítimas de violência psicológica do que as mulheres, o que não quer dizer, necessariamente, que as mulheres a pratiquem mais que os homens. 
Tabela 4.

Comparação por sexo nos escores da ECR-Brasil, ARI-O e ARI-E

\begin{tabular}{|c|c|c|c|c|c|c|c|c|}
\hline & Sexo & $\mathrm{N}$ & Média & $\begin{array}{l}\text { Desvio } \\
\text { Padrão }\end{array}$ & $\begin{array}{c}\text { Erro } \\
\text { Padrão }\end{array}$ & $\mathrm{t}$ & $\mathrm{gl}$ & $\mathrm{p}$ \\
\hline \multirow{2}{*}{ Evitação } & Feminino & 464 & 2,27 & 0,95 & 0,04 & \multirow{2}{*}{$\begin{array}{c}- \\
2,109\end{array}$} & \multirow{2}{*}{547} & \multirow{2}{*}{$0,035^{*}$} \\
\hline & Masculino & 85 & 2,51 & 0,99 & 0,11 & & & \\
\hline \multirow{2}{*}{ Ansiedade } & Feminino & 464 & 3,78 & 1,34 & 0,06 & \multirow{2}{*}{2,642} & \multirow{2}{*}{547} & \multirow{2}{*}{$0,008 *$} \\
\hline & Masculino & 85 & 3,36 & 1,39 & 0,15 & & & \\
\hline \multirow{2}{*}{$\begin{array}{c}\text { Atitudes } \\
\text { Controladoras - O }\end{array}$} & Feminino & 464 & 3,75 & 1,09 & 0,05 & \multirow{2}{*}{$\begin{array}{c}- \\
3,404\end{array}$} & \multirow{2}{*}{152,626} & \multirow{2}{*}{$0,001 *$} \\
\hline & Masculino & 85 & 4,08 & 0,77 & 0,08 & & & \\
\hline \multirow{2}{*}{$\begin{array}{c}\text { Atitudes } \\
\text { Controladoras - E }\end{array}$} & Feminino & 464 & 4,41 & 0,44 & 0,02 & \multirow{2}{*}{$\begin{array}{c}- \\
1,869\end{array}$} & \multirow{2}{*}{547} & \multirow{2}{*}{0,062} \\
\hline & Masculino & 85 & 4,50 & 0,40 & 0,04 & & & \\
\hline \multirow{2}{*}{ Físico - O } & Feminino & 464 & 1,33 & 0,64 & 0,03 & \multirow{2}{*}{4,159} & \multirow{2}{*}{209,034} & \multirow{2}{*}{$0,000 *$} \\
\hline & Masculino & 85 & 1,14 & 0,34 & 0,04 & & & \\
\hline \multirow{2}{*}{ Físico - E } & Feminino & 464 & 1,08 & 0,29 & 0,01 & \multirow{2}{*}{1,462} & \multirow{2}{*}{196,799} & \multirow{2}{*}{0,145} \\
\hline & Masculino & 85 & 1,05 & 0,16 & 0,02 & & & \\
\hline \multirow{2}{*}{ Psicológico - O } & Feminino & 464 & 2,15 & 0,98 & 0,05 & \multirow{2}{*}{2,028} & \multirow{2}{*}{157,022} & \multirow{2}{*}{$0,044^{*}$} \\
\hline & Masculino & 85 & 1,98 & 0,67 & 0,07 & & & \\
\hline \multirow{2}{*}{ Psicológico-E } & Feminino & 464 & 1,64 & 0,49 & 0,02 & \multirow{2}{*}{$\begin{array}{c}- \\
0,173\end{array}$} & \multirow{2}{*}{547} & \multirow{2}{*}{0,863} \\
\hline & Masculino & 85 & 1,65 & 0,45 & 0,05 & & & \\
\hline
\end{tabular}

Nota: $* \mathrm{p}<0,05$

Menores escores de violência psicológica indicam maiores níveis de violência.

Observando a Tabela 5, percebe-se elevadas correlações entre Atitudes Controladoras-O e Físico-O $(r=-0,639)$ indicando que quanto mais a pessoa percebe que sofre violência física, menos ela percebe que sofre atitudes controladoras e vice-versa; Atitudes Controladoras-O e Psicológico-O $(r=-0,728)$ indicando que pessoas que sofrem com atitudes controladoras, também percebem a violência psicológica (note-se que menores escores de violência psicológica, indicam maior violência sofrida) e por fim Físico-O com Psicológico-O $(r=0,552)$ indica que a pessoa que sofre com violência física tende a não sofrer com a violência psicológica. Em resumo, os dados mostram que a violência psicológica e as atitudes controladoras andam em um mesmo sentido, enquanto a violência física anda em sentido oposto.

O apego evitativo encontrou uma correlação fraca com as atitudes controladoras sofridas $(r=-0,332)$. Ao mesmo tempo, a ansiedade correlacionou-se com as atitudes controladoras $(r=-0,307)$ e a violência psicológica praticadas pelo participante $(r=0,380)$, no sentido de que uma maior ansiedade está associada com a realização de poucas atitudes 
controladoras e pouca violência psicológica. Não foram encontradas correlações de grande magnitude com a idade.

Tabela 5.

Correlação de Pearson entre idade e a ECR-Brasil, ARI-O e ARI-E

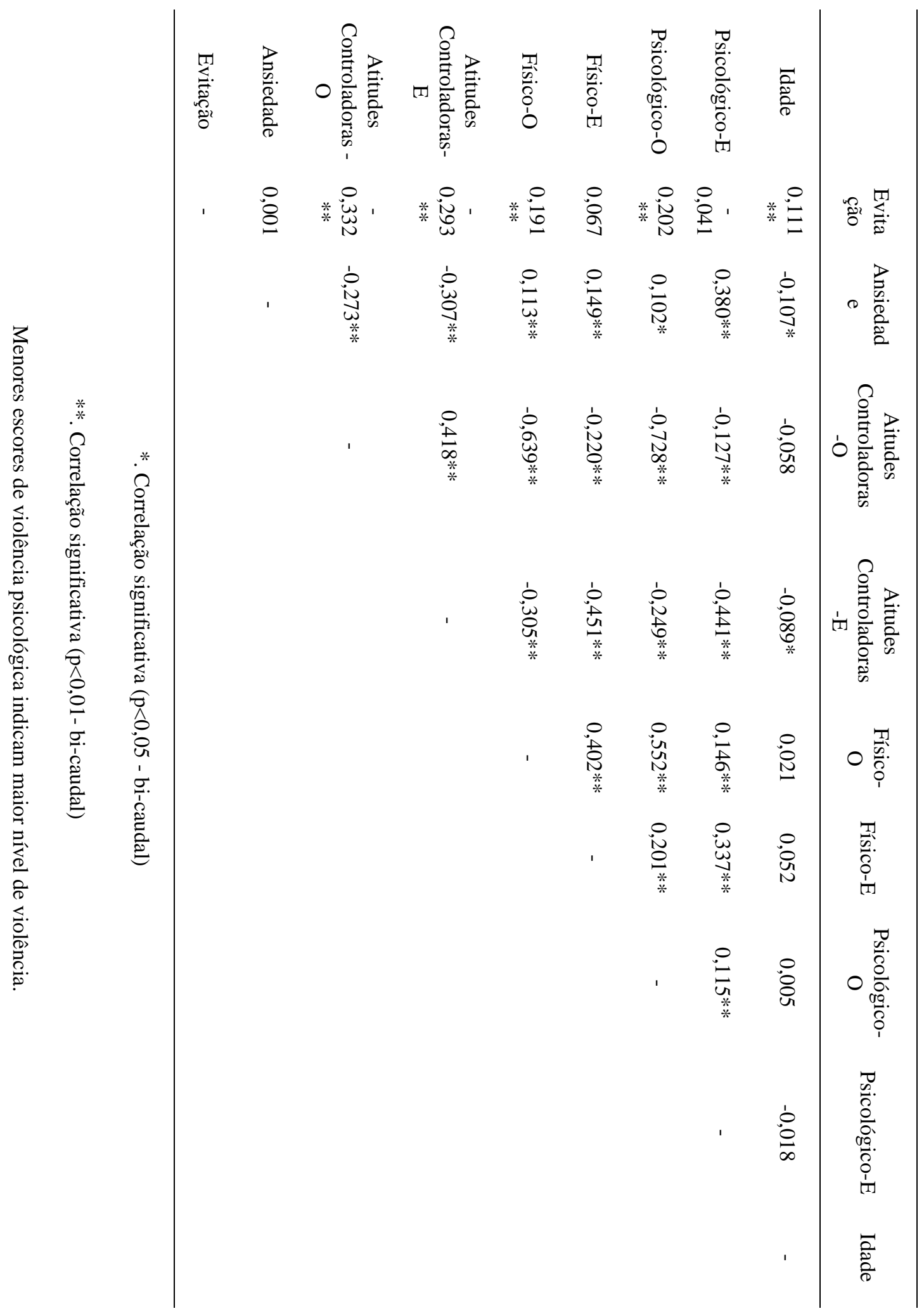




\section{Discussão}

Com a conscientização acerca da violência sobre a mulher como degradação dos princípios básicos dos direitos humanos, aumentou-se a percepção da manutenção dessas práticas em diferentes locais ao longo do mundo. O "despertar" social dessa temática reafirma a relevância em se pesquisar e trazer a luz dos fatos a estruturação sócio-histórica e científica da violência sofrida pelas mulheres dentro de relacionamentos íntimos. Esse "despertar" se dá como consequência do novo posicionamento da mulher perante a sociedade e ela mesma, provocado pela maior transparência nas relações interpessoais, movimentos públicos e políticos, rede de apoio às vítimas e a própria manifestação destas sobre as violências que sofrem.

Somente a partir dos anos noventa, uma série de fatores despertaram o interesse a respeito da temática sobre relacionamentos abusivos. Dentre esses fatores, estão a necessidade de desenvolver novas ferramentas teóricas que permitissem aumentar a sua compreensão e a sua abordagem; a necessidade do desenvolvimento de novas metodologias de aproximação ao objeto que permitissem "registrá-lo" e caracterizá-lo, culminando em uma ampla gama de publicações acerca do tema (Matos, 2006).

Esse estudo evidenciou boas propriedades para as escalas ARI-E e ARI-O. Dada a escassez de instrumentos de medida na área da violência em relacionamentos íntimos, este estudo apresenta uma ferramenta importante na detecção desse problema social. Mesmo que a estrutura fatorial precise ser lapidada em outros estudos, a escala ARI é precisa e tem boas propriedades psicométricas. Assim, este estudo colabora com a crescente tendência de se investigar instrumentos associados a relacionamentos no Brasil (Cassepp-Borges \& De Andrade, 2013).

Os itens que tiveram uma carga fatorial muito diferente na ARI-O e ARI-E foram 1, 2, 3, 4, 5 no fator Violência Psicológica e 8 e 11 no fator Violência Física. Nota-se que os itens invertidos da sub-escala da violência psicológica tiveram uma carga fatorial forte com o seu fator na ARI-O, o que não foi repetido na ARI-E. Isso significa que esses itens se refiram a uma violência psicológica somente quando a pessoa sofre a violência, mas não quando a pessoa pratica a violência. Como exemplo temos o item 2. Quando se refere a um comportamento do outro para com o respondente ("Incentiva-me a tentar coisas novas") ele é associado ao inverso da violência psicológica. Quando é um comportamento 
do respondente para com o outro (“Incentivo a tentar coisas novas") não está associado a uma violência psicológica.

O fato é que a escala ARI precisa de aprimoramentos, a fim de que possa ter a sua invariância demonstrada. Entretanto, esse problema pode se referir não a uma distorção na escala, mas a uma distorção no próprio comportamento dos participantes, que vem a estrutura fatorial da violência que sofrem como diferente da estrutura fatorial da violência que praticam. A violência psicológica é tão sutil que, quando praticada pelo respondente, ela não é percebida como uma violência. Mas quando o respondente sofre, isso é violento e tem algum impacto. Com relação a atitudes controladoras, percebeu-se que os itens 8 e 11 se referem a questão de vestimenta apenas são considerados atitudes controladoras quando o respondente sofre esse comportamento (ARI-O). Pode-se hipotetizar que isso tenha relação com o fato da amostra ser composta por $84,5 \%$ de mulheres. Controlar a vestimenta, por exemplo, é um comportamento mais masculino do que feminino, como aponta Marques (2005).

Apesar do grande volume de publicações sugerindo uma violência por parte dos homens em relação à mulher (Matos, 2006; Marques, 2005; Miranda, 2010; Caridade \& Machado, 2006, Saffioti, 2004), este estudo sugere que isso é verdadeiro somente em relação à violência física. As atitudes controladoras e violência psicológica parecem ser mais praticadas pelo sexo feminino do que pelo masculino. Entretanto, há uma restrição nesse dado, pois a amostra foi escolhida por conveniência. Acredita-se que pessoas que sofrem quaisquer tipo abuso, independente do gênero, têm uma maior tendência a responder a um questionário sobre o tema. Assim, a amostra de homens e mulheres que responderam o questionário pode não ser representativa do universo de homens e mulheres na sociedade. Mesmo assim, além da questão de gênero, futuros estudos podem aprofundar-se na questão da violência conjugal entre pessoas de diferentes orientações sexuais.

A partir dos resultados encontrados nas correlações de Pearson, percebeu-se uma leve influência do apego em relação à violência. A correlação negativa entre o apego evitativo e atitudes controladoras sofridas pode ser explicada pelo fato de que as pessoas que possuem apego evitativo tendem a se distanciar de pessoas controladoras. Ao mesmo tempo, pessoas com apego ansioso tendem a praticar menos violência psicológica e atitudes controladoras. Uma hipótese para isso é a de que pessoas com apego ansioso sintam algum medo de perder o seu parceiro e por isso controlem mais esses impulsos. 
Embora haja, neste estudo, a correlação estatística entre os diferentes tipos de abusos e violências em busca do entendimento de sua origem, a abordagem estatística inviabiliza o entendimento da contextualização da relação entre vítima e abusador. Abordagens quantitativas desconsideram fatores circunstanciais acerca do evento, tais como as causas e razões que levaram à ocorrência, possíveis sequências de atos entre os envolvidos e a percepção do abuso propriamente dito pela ótica da vítima.

A violência de gênero deve ser compreendida em seus diversos aspectos, para uma análise ampla (Saffioti, 2004). Por meio desta ótica, nota-se que há muitas opções de atuação dentro da Psicologia em função da redução de transtornos causados dentro de relacionamentos abusivos, considerando que muitas das relações de possíveis causas e efeitos potenciais são vastamente estudados por esta área do conhecimento. Seja com atuação direta acompanhando o casal no entendimento da relação em clínicas particulares ou recebendo pessoas que necessitem de ajuda em locais especializados em apoio à vítima, tal qual em uma Delegacia da Mulher, o auxílio profissional de um psicólogo prova ser essencial em casos de violência conjugal. Espera-se que este trabalho possa contribuir para o alinhamento entre teoria e prática durante a busca pela compreensão de comportamentos descritos e suas origens, tornando-se suporte auxiliar aos profissionais analíticos e/ou pesquisadores focados no tema. 


\section{Referências}

Aldrighi, T. (2004). Prevalência e cronicidade da violência física no namoro entre jovens universitários do estado de São Paulo. Psicologia, Teoria e Prática, 6(1), 105-120.

Anacona, C. A. (2008). Prevalencia, factores de riesgo y problemáticas asociadas con la violencia en el noviazgo: una revisión de la literatura. Avances en Psicología Latinoamericana, 26(2), 227-241.

Barnett, O. W., Miller-Perrin, C. L., \& Perrin, R. D. (1997). Family violence across the lifespan. Sage Publications.

Brennan, K., Clark, C. L., \& Shaver, P. R. (1998). Selfreport measurement of adult attachment: an integrative overview. In J.A. Simpson \& P.R. Shaver (1985). Attachment theory and close relationships." (p. 46-76). Guilford Press.

Cassepp-Borges, V., \& De Andrade, A. L. (2013). Uma breve história das tentativas para medir atributos dos relacionamentos amorosos em língua portuguesa. Estudos de Psicologia (Natal), 18(4), 631-638. https://doi.org/10.1590/S1413-294X2013000400011

Caridade, S., \& Machado, C. (2006). Violência na intimidade juvenil: da vitimação à perpetração. Análise Psicológica, 4(24), 485-493. https://doi.org/10.14417/ap.541

Chan, K. L., Straus, M. A., Brownridge, D. A., Tiwari, A., \& Leung, W. C. (2008). Prevalence of dating partner violence and suicidal ideation among male and female university students worldwide. Journal of Midwifery \& Womens Health, 53(6), 529-537. https://doi.org/10.1016/j.jmwh.2008.04.016

Cornelius, T. L., \& Resseguie, N. (2007). Primary and secondary prevention programs for dating violence: a review of the literature. Aggression and Violent Behavior, 12(3), 364-375. https://doi.org/10.1016/j.avb.2006.09.006

Dilillo, D., Giuffre, D., Tremblay, G. C., \& Peterson, L. (2001). A closer look at the nature of intimate partner violence reported by women with a history of child sexual abuse. Journal of Interpersonal Violence, 16(2),116-132. https://doi.org/10.1177/088626001016002002

Flake, T. A., Barros, C., Schraiber, L. B., \& Menezes, P. R. (2013). Violência por parceiro íntimo entre estudantes de duas universidades do Estado de São Paulo, Brasil. Revista Brasileira de Epidemiologia, 16(4), 801-816. https://doi.org/10.1590/S1415-790X2013000400001

Hazan, C., \& Shaver, P. (1987). Romantic love conceptualized as an attachment process. Journal of Personality and Social Psychology, 52(3), 511-524. https://doi.org/10.1037/0022-3514.52.3.511

Henderson, A. J. Z., Bartholomew, K., Trinke, S.J., \& Kwong, M. J. (2005). When loving means hurting: An exploration of attachment and intimate abuse in a community sample. Journal of Family Violence, 20(4), 219-230. https://doi.org/10.1007/s10896-005-5985-y

Hickman, L. J., Jaycox, L. H., \& Aronoff, J. (2004). Dating violence among adolescents: prevalence, gender distribution, and prevention program effectiveness. Trauma, Violence \& Abuse, 5(2), 123-142. http://doi.org/10.1177/1524838003262332

Hohendorff, J. V., Habigzang, L. F., \& Koller, S. H. (2007). A boy, being a victim, nobody really buys that, you know?': Dynamics of sexual violence against boys. Child Abuse \& Neglect, 70, 53-64. https://doi.org/10.1016/j.chiabu.2017.05.008 
Hohendorff, J. V., Santos, S. S., \& Dell'Aglio, D. D. (2015). Estudo de caso sobre a revelação da violência sexual contra meninos. Contextos Clínicos, 8, 46-54. https://doi.org/10.4013/ctc.2015.81.05

Krug, E. G, Dahlberg, L. L., Mercy, J. A., Zwi. A. B. \& Lozano, R. (2002). World report on violence and health. Geneva: World Health Organization. https://doi.org/10.1016/S0140-6736(02)11133-0

Love is Respect (2016). Are you in a healthy relationship? Disponível em http://www.loveisrespect.org/for-someone-else/is-my-relationship-healthyquiz/

Marques, T. M. (2005). Violência conjugal: estudo sobre a permanência da mulher em relacionamentos abusivos. Dissertação de Mestrado não publicada Universidade Federal de Uberlândia, Uberlândia, Minas Gerais. Disponível em https://repositorio.ufu.br/handle/123456789/17261

Matos, M. A. V. (2006). Violência nas relações de intimidade: estudo sobre a mudança psicoterapêutica na mulher. Tese de Doutorado em Psicologia. Disponível em http://hdl.handle.net/1822/5735

Miranda, M. P. M., Paula, C. S., \& Bordin, I. A. (2010). Violência conjugal física contra a mulher na vida: prevalência e impacto imediato na saúde, trabalho e família. Revista Panamericana de Salud Pública, 27(4), 300-308. https://doi.org/10.1590/S1020-49892010000400009.

Murta, S. G, Santos B. R. P., Martins, C. P. S., \& Oliveira, B. (2013). Prevenção primária à violência no namoro: uma revisão de literatura. Contextos Clínicos, 6(2), 117-131. http://doi.org/10.4013/ctc.2013.62.05.

Natividade, J. C., \& Shiramizu, V. K. M. (2015). Uma medida de apego: versão brasileira da Experiences in Close Relationship Scale-Reduzida (ECR-RBrasil). Psicologia USP, 26(3), 484-494. https://doi.org/10.1590/0103$\underline{656420140086}$

Oliveira, Q. B. M., Assis, S. G., Njaine, K., \& Oliveira, R.V.C. (2011). Violência nas relações afetivo-sexuais. In: C. M. Minayo, S. G. Assis, \& K. Njaine: Amor e violência: um paradoxo das relações de namoro e do 'ficar' entre jovens brasileiros (pp. 87-141). Fiocruz.

Paiva, C., \& Figueiredo, B. (2003) Abuso no Relacionamento Íntimo com o Companheiro: Definição, Prevalência, Causas e Efeitos, Psicologia, Saúde \& Doenças, 4(2), 165-184.

Paiva, C., \& Figueiredo, B. (2004). Abuso no relacionamento íntimo: estudo da prevalência em jovens adultos portugueses. Psychologica, 36, 75-107.

Piosiadlo L. C. M., Fonseca, R. M. G. S., \& Gessner, R. (2014). Subalternidade de gênero: refletindo sobre a vulnerabilidade para violência doméstica contra a mulher. Escola Anna Nery, 18(4), 728-733. https://doi.org/10.5935/1414$\underline{8145.20140104}$

Saffioti, H. (2004) Gênero, patriarcado, violência. Fundação Perseu Abramo

Ting, S. R. (2009). Meta-analysis on dating violence prevention among middle and high schools. Journal of School Violence, 8(4), 328-337. http://doi.org/10.1080/15388220903130197

Williams, J. R., Ghandour, R. M., \& Kub, J. E. (2008). Female perpetration of violence in heterosexual intimate relationships. Trauma, Violence \& Abuse, 9(4), 227-249. http://doi.org/10.1177/1524838008324418 